\title{
L'évolution des primes embarrasse peu la population
}

\section{Jürg Schlup}

Dr méd., président de la FMH

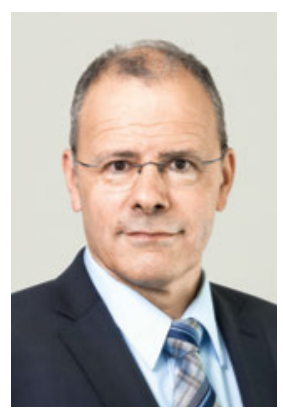

«Les primes explosent le budget de près d'une famille sur deux» titrait en août le journal 20 minutes en Suisse alémanique et au Tessin en citant le site internet de Comparis. Nombre de lecteurs ont dû être irrités, d'autant plus que l'information principale, au sujet des données utilisées, ne leur a pas été donnée: bien caché en bas de la page du site de Comparis, un texte anodin précise que l'enquête «n'est pas représentative» [1]. Le contraire aurait été étonnant au vu des chiffres des enquêtes représentatives, qui donnent une tout autre image: la part de la population qui considère que les primes d'assurance-maladie sont un problème est descendue en 2017 à son niveau le plus bas depuis 15 ans [2-3]. La part qui considère les coûts médicaux (méde-

\section{La part de la population qui considère que les} primes d'assurance-maladie sont un problème est à son niveau le plus bas depuis 15 ans.

cins et médicaments) comme un problème a même été presque divisée par deux l'année dernière [2]. Bien évidemment, le fait qu'aujourd'hui un tiers des personnes estime toujours que les primes sont un problème [2] impose de trouver des solutions. Mais restons dans le contexte: un tiers de la population [2], d'autres enquêtes indiquent même la moitié [4], classe la charge fiscale au premier rang des dépenses qui pèsent lourdement sur le budget des ménages. Et pourtant, la discussion sur le plafonnement des coûts se porte exclusivement sur les prestations de santé - pourtant vitales - et non sur le budget de l'administration publique par exemple.

Cela ne correspond pas aux préférences de la population: si elle pouvait réorganiser les dépenses de l'Etat, elle diminuerait p.ex. le budget de l'administration publique de $24 \%$ mais, en revanche, elle augmenterait les dépenses de la santé de $8 \%$ [4]. Cette augmentation est judicieuse, car la majorité de la population aimerait dépenser au moins autant d'argent pour tous les domaines de la santé sans exception et elle rejette clairement la limitation des traitements très onéreux, qu'elle soit proposée par le Tribunal fédéral (65\%), l'OFSP (76\%) ou les caisses-maladie (78\%) [3].
En matière de consommation, la population adopte un comportement différencié: la plupart reconnaît la nécessité d'une meilleure prise de conscience des coûts, comme le montre l'approbation croissante de la hausse des franchises minimale (53\%) et maximale (58\%) [2]. La population se préoccupe de la répartition des charges, comme l'indiquent les majorités réunies en faveur de l'exonération des primes pour enfants en particulier pour les ménages à faibles revenus - et de leur diminution pour les jeunes adultes [2].

Mais de telles mesures ponctuelles ne sont pas le seul moyen d'encourager la responsabilité de chacun, de sensibiliser aux questions de coûts, de mieux répartir les charges et de soulager les payeurs de primes. La mission la plus urgente de la politique de la santé serait de faire avancer le dossier du financement uniforme des prestations ambulatoires et hospitalières. Sinon, le transfert des traitements hospitaliers - financés principalement par les cantons - vers le secteur ambulatoire - exclusivement financé par les primes - sera toujours plus à la charge des assurés. Rien que cette correction des incitatifs négatifs, déterminés aujourd'hui par la répartition du financement, révélerait selon différentes projections un important potentiel d'économie - sans rationnement ni perte de qualité [5].

\section{Si la population pouvait réorganiser les} dépenses de l'Etat, elle augmenterait les dépenses de santé de $8 \%$.

Si les bonnes nouvelles étaient autant attendues que les titres à scandale, la manchette de 20 minutes aurait pu être la suivante «L'évolution des primes embarrasse peu la population»: les primes ne comptaient plus qu'à hauteur de 21\% en 2016 parmi les inquiétudes les plus importantes des Suisses, contre 63\% en 2003 [6]. Il aurait également été correct de dire que «quatre personnes sur cinq trouvent que l'évolution du système de santé est positive» - et de souligner que ce taux est passé de $47 \%$ en 2003 à $81 \%$ [3]. Il est clair que nous devons trouver des solutions aux problèmes et favoriser les améliorations. En revanche, les vents de panique ne sont jamais de bons conseillers! 